\title{
Antibiotic Prescription Pattern for Neonatal Sepsis at the University College Hospital, Ibadan; How Judicious
}

\author{
Olukemi Oluwatoyin Tongo ${ }^{1,2,}$, Adeyemi Adeyinka Labaeka ${ }^{2}$ \\ ${ }^{1}$ Department of Pediatrics, University of Ibadan, Ibadan, Nigeria \\ ${ }^{2}$ Department of Pediatrics, University College Hospital, Ibadan, Nigeria
}

Email address:

ktongo2@yahoo.com (O. O. Tongo), yemilabaeka2@gmail.com (A. A. Labaeka)

${ }^{*}$ Corresponding author

\section{To cite this article:}

Olukemi Oluwatoyin Tongo, Adeyemi Adeyinka Labaeka. Antibiotic Prescription Pattern for Neonatal Sepsis at the University College Hospital, Ibadan; How Judicious. American Journal of Pediatrics. Vol. 6, No. 1, 2020, pp. 58-61. doi: 10.11648/j.ajp.20200601.20

Received: February 3, 2020; Accepted: February 14, 2020; Published: February 26, 2020

\begin{abstract}
Multidrug-resistant bacterial infection is a global phenomenon and newborn units are not spared. Judicious use of antibiotics is one of the measures employed to curb the emergence of multidrug-resistant bacteria. It entails the use of empiric antibiotics based on knowledge of prevailing pathogens and antibiotic susceptibility patterns while awaiting the results of cultures. This retrospective review of records of 170 outborn neonates treated at the emergency room of the University College Hospital (UCH), Ibadan sought to determine indications for antibiotic therapy, the available supporting investigations and factors militating against the judicious use of antibiotics in newborns. The median age at presentation was three days. The majority (91.2\%) were commenced on antibiotics on admission without any prior laboratory investigations. The indications for antibiotics were based on clinical signs only in $48 \%$ of cases, risk factors for sepsis with clinical signs in $42 \%$ of cases and no apparent reason in $6.8 \%$. Lack of funds and logistic problems with the laboratory were the reasons for not conducting investigations before the commencement of antibiotics in $49.4 \%$ and $15.3 \%$ of cases respectively. Full blood count was eventually done in $32.9 \%$ of which a third were consistent with sepsis. Blood cultures were eventually done in $33.5 \%$ and positive in $5.3 \%$ of cases. Majority of newborns had empiric antibiotic therapy without the necessary laboratory back up due largely to financial constraints and other logistic issues with the laboratory. Inappropriate use of antibiotics in terms of initiation of empiric treatment, choice of drugs and failure to investigate as necessary was common.
\end{abstract}

Keywords: Neonatal Sepsis, Judicious, Rational Antibiotics

\section{Introduction}

Neonatal infections remain a major cause of neonatal mortality in developing countries, its clinical signs are subtle and nonspecific but with severe consequences. [1-4] In view of the negative impact of infections on mortality and long term outcomes, empirical antibiotics are usually administered to symptomatic newborns or those at high risk of sepsis while awaiting culture results, with subsequent continuation or discontinuation guided by of culture and sensitivity results. [5] The indiscriminate and prolonged use of broad-spectrum antibiotics (especially $3^{\text {rd }}$ generation cephalosporins) in newborns has been associated with necrotizing enterocolitis, late-onset sepsis and invasive candidiasis. [6] The menace of multidrugresistant bacteria has been of great public health concern globally, particularly in developing countries [2, 7], yet the prospect of new classes of antibiotics emerging soon is slim [8]. The unrestricted use of broad-spectrum antibiotics is reported to be a contributory factor to the emergence of drug resistance. Judicious use of antibiotics refers to the use of antibiotics in a manner that will eradicate bacteria, optimize treatment by diagnosis and severity assessment taking into cognizance the prevalence of local resistance, pharmacokinetics and pharmacodynamics, dosage, efficacy and cost effectiveness. [9] It is therefore, one of the control measures required in the newborn unit to curb the emergence of multidrug-resistant bacteria.

\section{Materials and Methods}

This was a retrospective review of the case records of 170 outborn neonates admitted into the Children's Emergency 
Ward of the University College Hospital, Ibadan over a 6month period. At this time, there was no written antibiotic policy in the unit but there was an unwritten rule to use Ampicillin/Sulbactam and amikacin as the first-line antibiotic for neonatal sepsis.

The objective of this study was to determine how rational the prescription of antibiotics was, among referred newborns presenting at the University College Hospital (UCH), Ibadan.

The information extracted included the demographic details, presenting features, presumptive diagnosis, investigations requested and those carried out, where investigation was not carried out, the reasons given, antibiotics were given, duration and the time of availability of results were entered into IBM SPSS statistical package version 20.0, all analysis and calculations were done using the same statistical package. Data were presented as mean $\pm \mathrm{SD}$ and median for continuous variables with normal and skewed distribution respectively and as proportions for categorical variables.

\section{Results}

\subsection{General Characteristics of Patients}

The mean age of the babies on admission was $4.82+5.5$ days, with a median of 3 days. Their mean gestational age was $37 \pm 3$ weeks. The sex distribution showed 88 (51.8\%) were males and $82(48.2 \%)$ females (M: F ratio 1.1:1). The babies were referred from different sources as shown in figure 1, with private hospitals being the source of referral in $71(41.8 \%)$ cases. All investigations and medications were paid for from out of pocket by babies' families on cash and carry basis.

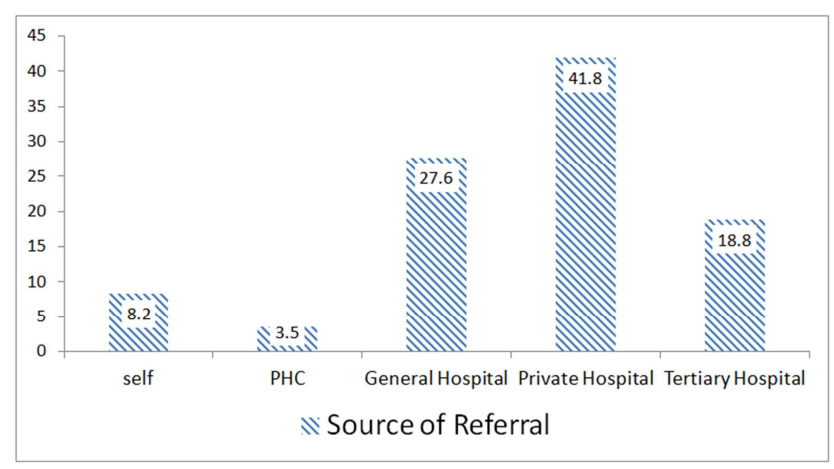

Figure 1. Sources of referral.
On admission, $20(11.8 \%)$ babies had no presumptive diagnosis of sepsis or identifiable risk factors for sepsis. A presumptive diagnosis of early-onset sepsis (EOS) and lateonset sepsis (LOS) was made in $111(65.3 \%)$ and $39(22.9 \%)$ of cases respectively.

One hundred and sixty-one (94.7\%) were commenced on antibiotics on admission, but only 150 (93\%) were justifiable in terms of presence of clinical signs or risk factors for sepsis. There was no apparent reason for initiation of antibiotic therapy in $11(7 \%)$ of cases as shown in figure 2 .

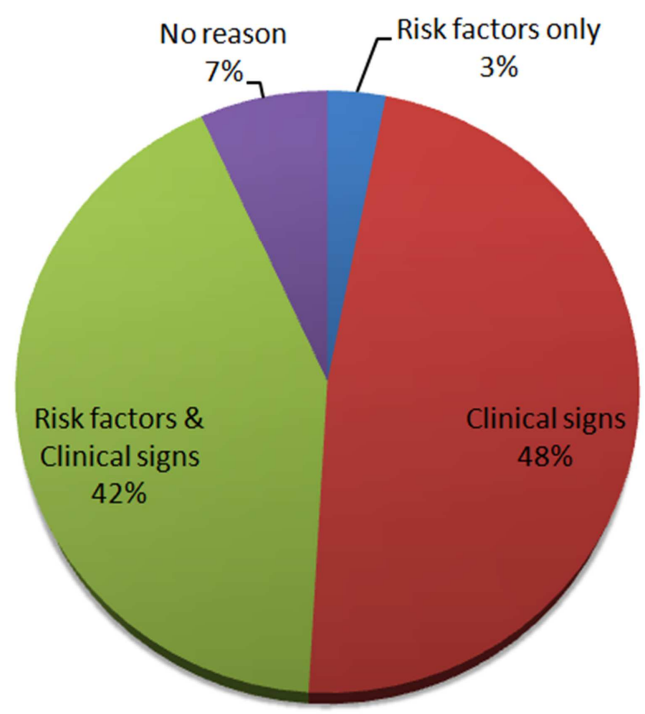

Figure 2. Indications for initiating antibiotics.

Only $6(3.5 \%)$ newborns had any laboratory investigations done before the commencement of antibiotics while 155 (91.2\%) newborns were commenced on antibiotics before any laboratory investigations were carried out and 9 (5.3\%) were not commenced on antibiotics at all. The reasons for not investigating before the commencement of antibiotics were; no funds 84 (49.4\%), logistic problems in the laboratory 26 $(15.3 \%)$ and investigations not requested in $45(26.5 \%)$ of cases.

More than three quarters $133(78.2 \%)$ eventually had any laboratory investigations for sepsis at one time or the other during the course of antibiotic treatment. The investigations done and outcomes are as shown in table 1 .

Table 1. Investigations done and outcome of results.

\begin{tabular}{|c|c|c|c|c|}
\hline \multicolumn{5}{|l|}{ Results } \\
\hline Investigation & Number requested $(\%) \mathrm{N}=161$ & Consistent with sepsis (\%) & Not suggestive of sepsis (\%) & Results not available (\%) \\
\hline$\mu \mathrm{ESR}$ & $102(63.4)$ & $36(35.3)$ & $66(64.7)$ & - \\
\hline Full blood count & $56(34.8)$ & $20(35.7)$ & $30(53.6)$ & $6(10.7)$ \\
\hline Blood culture & $57(35.4)$ & $9(15.8)$ & $19(33.3)$ & $29(50.9)$ \\
\hline CSF Cultures & $44(27.3)$ & $4(9.1)$ & $38(86.4)$ & $2(4.5)$ \\
\hline
\end{tabular}

Of those who had investigations done, full blood count results were available within 24 hours in $60.7 \%$, 48hours in $19.6 \%$, after 48 hours in $8.9 \%$ of cases. More than half of the blood culture results were not available till discharge. The available blood culture results, were available within 3 days in $1.8 \%, 4$ days in $3.5 \%, 5$ days in $8.8 \%, 7$ days in $22.8 \%$ and $>7$ days in $12.3 \%$ of cases.

Lumbar puncture was not indicated in $126(74.1 \%)$ of cases. Of the CSF analyses that were done, preliminary results were available within 24 hours in $95.4 \%$ of cases and 
full culture results available after 72 hours in those cases.

\subsection{Administration of Antibiotics}

All antibiotics were administered intravenously. A combination of Ampicillin/Sulbactam and amikacin was used as the first-line empirical therapy in $130(80.7 \%)$ cases as shown in table 2. Antibiotic administration was regular in $135(83.9 \%)$ and not regular in $26(16.1 \%)$ of cases. Of the cases of irregular administration of antibiotics, $14(53.8 \%)$ were due to difficulties with vascular access while the rest had to do with non-provision of the drugs by parents due to financial constraints.

Table 2. Choice of first line/empirical antibiotics in 161 babies.

\begin{tabular}{lll}
\hline Type of antibiotics & Frequency (n) & Proportion (\%) \\
\hline Amp/Sulbact \& Amikacin & 130 & 80.7 \\
Cefuroxime \& Gentamycin & 8 & 5.0 \\
Cefotaxime & 1 & 0.6 \\
Ceftazidime \& Gentamycin & 18 & 11.2 \\
Ceftriaxone \& Gentamycin & 4 & 2.5 \\
\hline
\end{tabular}

In $58(36 \%)$ cases, the babies required a change of antibiotics to second-line drugs, 51 (88\%) of which had no laboratory back up before the change. One child had to be changed to oral drugs because of difficulties with vascular access. The indications for changing to second-line are as shown in table 3.

Table 3. Indications for change of antibiotics to second-line.

\begin{tabular}{ll}
\hline Indications & Frequency n (\%) $\mathbf{( N = 5 8 )}$ \\
\hline None apparent & $8(13.8)$ \\
No clinical response & $15(25.8)$ \\
Clinical deterioration after initial response & $33(56.9)$ \\
Financial consideration & $2(3.4)$ \\
Difficulties with vascular access & $1(1.7)$ \\
\hline
\end{tabular}

\section{Discussion}

Neonatal sepsis can be rapidly fatal hence it is standard practice to commence empiric antibiotic therapy in suspected cases, after prompt sepsis screening while awaiting results of investigations in order to prevent fatality. [8] Such empiric antibiotics are usually decided based on the knowledge of prevailing organisms and antibiotic susceptibility pattern in that setting. The prevalence of antibiotic use among newborns without the necessary laboratory workup prior to treatment in this study was however very high. Over ninety percent of the babies were commenced on antibiotics prior to investigations while only $3.5 \%$ of the babies had any investigations done before the commencement of antibiotics. In others who had the investigations eventually carried out after commencement of antibiotics, the results may have been modified by the use of antibiotics such that it will be difficult to conclude if such babies had the infections at the onset or not hence taking a decision to discontinue treatment becomes difficult further promoting overuse of antibiotics. This cannot be described as judicious use of antibiotics. As seen in this study, only $15.8 \%$ of the blood cultures eventually taken were positive and about a third of full blood counts done were consistent with sepsis yet over $90 \%$ of the babies were treated with antibiotics. The antibiotic pressure seen in this study borne as a result of inadequate laboratory back up has the potential of promoting antibiotic resistance in an already resource-constrained environment where facilities are few and there are limited affordable antibiotics to choose from. [10, 11] The use of antibiotics in infants without culture-proven sepsis has been reported to lead to increased antibiotic use with attendant adverse outcomes. [12] Majority of these cases that did not have investigations prior to initiation of antibiotics had to do with financial constraints on the part of parents and logistic issues in the laboratory.

Out of pocket healthcare financing as it operates in this setting puts families of dependent populations like newborns at higher risk of catastrophic health expenditure, further worsening the level of poverty [13]. Also, caring for these sick babies by direct out of pocket spending leaves them at risk of not receiving the much-needed care if the parents are unable to provide the necessary funds. An earlier study on the economic cost of preterm very low birth weight care from our centre reported that parents spent $22.8-3966 \%$ of their combined monthly family income on the care of their babies during neonatal admission. [14] In order to mitigate against these problem, it is essential to find alternative means of financing newborn care other than directly out of pocket of the parents. It is also crucial to ensure that laboratories are functioning optimally to provide the necessary support for diagnosis of infections in newborns to curb further non-judicious use of antibiotics.

It is also noteworthy that a proportion of the babies had no justifiable indications for antibiotics either in terms of clinical signs or presence of risk factors, barely a third had blood cultures done with $15.8 \%$ of those done being positive. Also, among those that had full blood count done, barely a third were consistent with sepsis though reliance on full blood count for the diagnosis of infection in the newborn is contentious, but it suggests that continued antibiotic treatment was probably not indicated in the majority. Inappropriate use of antibiotics in terms of initiation and discontinuation in this population was quite prevalent when compared with developed countries. $[15,16]$ This has the potential of predisposing to multidrug-resistant gramnegative bacterial infections in the hospital with the possibility of transmission to the community. [2, 12, 17] It will also contribute to increased cost of care and prolonged hospital stay in an already overburdened healthcare system.

A substantial proportion of babies were started on $3^{\text {rd }}$ generation cephalosporin as the first-line with the attendant risk of adverse outcomes. [6] Having a written sepsis screening and antibiotic protocol as practiced in developed countries could also have prevented this [7].

It has been well documented that adequate laboratory back up is lacking in many developing countries and this is also evident in the limited spectrum of diagnostic/supportive investigations available in this study, which were not even adequately utilized. In some situations, results of these investigations were never available to guide treatment even till death or discharge. The 
irregularity of the antibiotics was another observed challenge, which is a potential contributor to the emergence of antimicrobial resistance. More than a third of the babies were changed to second-line drugs but again majority were clinical decisions without any laboratory back up for such changes. The choice of first-line antibiotics was Ampicillin/Sulbactam and amikacin in about $76.5 \%$ of cases, which was in conformity with the findings of Akindolire et al [18] on neonatal sepsis in the same hospital though there were no written guidelines in place at the time of the study. A substantial number were started on $3^{\text {rd }}$ generation cephalosporins and gentamicin as the first-line and this has the potential of predisposing to HAI with multidrug-resistant organisms with little choices of antibiotics to change to if need be. Having written antibiotic guidelines is therefore expedient.

\section{Conclusion}

The use of empiric antibiotics in sick neonates without laboratory support is prevalent. Point of care user fees and other logistic issues within the hospital contributed to this practice. It is recommended that written sepsis screen protocols and antibiotic prescription guidelines be instituted while the care of newborns should not depend on out of pocket point of care user fees in hospitals in order to promote the judicious use of antibiotics.

\section{Disclosures}

The authors did not receive any funding for this research but the study was carried out as a form of a clinical audit of practice.

\section{Conflict of Interest}

The authors did not have any conflict of interest.

\section{References}

[1] World Health Organization. Neonatal and Perinatal Mortality: Country, regional and global estimates. 2006.

[2] Vergnano S, Sharland M, Kazembe P, Mwansambo C, Heath PT. Neonatal sepsis: an international perspective. Arch. Dis. Child. Fetal Neonatal Ed. 2005; 90: 220-224.

[3] Stoll BJ, Hansen NI, Adams-Chapman I, et al. Neurodevelopmental and growth impairment among extremely low-birth-weight infants with neonatal infection. JAMA. 2004; 292 (19): 2357-2365.

[4] Klinger G, Levy I, Sirota L, et al. Outcome of early-onset sepsis in a national cohort of very-low-birth-weight infants. Pediatrics. 2010; 125 (4): e736-e740.

[5] Clark RH, Bloom BT, Spitzer AR, et al. Reported medication use in the neonatal intensive care unit: data from a large national data set. Pediatrics. 2006; 117 (6): 1979-1987.
[6] Tripathi N, Cotton CM, Smith PB. Antibiotic use and misuse in the Neonatal Intensive Care Unit. Clinics in Perinatology 2012; 39 (1): 61-68.

[7] Carlet J, Rambaud C and Pulcini C. Save Antibiotics: a call for action of the World Alliance Against Antibiotic Resistance (WAAAR). BMC Infectious Diseases. 2014, 14: 436 available at http://www.biomedcentral.com/1471-2334/14/436.

[8] Piddock L J: The crisis of no new antibiotics-what is the way forward. Lancet Infect Dis 2012, 12: 24953.

[9] Ball P1, Baquero F, Cars O, File T, Garau J, et al. Antibiotic therapy of community respiratory tract infections: strategies for optimal outcomes and minimized resistance emergence. Journal of Antimicrobial Chemotherapy 2002; 49: 31-40.

[10] Society for Healthcare Epidemiology of America, Infectious Diseases Society of America, Pediatric Infectious Diseases Society. Policy statement on antimicrobial stewardship. Infect Control Hosp Epidemiol 2012; 33 (4): 322-327.

[11] Dellit JH, Owens RC, Jr McGowan JE, Gerding DN, Weinstein RA, Burke JP, Hushing WC, Paterson DL, Fishman NO, Carpenter CF, Brennan PJ, Billeter M, Hooton TM. Infectious diseases society of America and society of health care epidemiology of America guidelines for developing an institutional program to enhance antibiotic stewardship. Clin Infect Dis 2007, 44: 159-77.

[12] Ting JY, Synnes A, Roberts A, Deshpandey A, Dow K, Yoon EW, Lee KS, Dobson S, Lee SK, Shah PS; Canadian Neonatal Network Investigators. Association Between Antibiotic Use and Neonatal Mortality and Morbidities in Very Low-BirthWeight Infants Without Culture-Proven Sepsis or Necrotizing Enterocolitis. JAMA Pediatr. 2016; 170 (12): 1181-1187.

[13] Aregbeshola BS. Out-of-pocket payments in Nigeria. The Lancet 2016; 387 (10037): 2506.

[14] Tongo O O., Orimadegun A E, Ajayi S O, Akinyinka O O. (2009) The Economic Burden of Preterm/Very Low Birth Weight Care in Nigeria. Journal of Tropical Paediatrics. Vol. 55. No. 4. 262-264.

[15] Patel SJ, Oshodi A, Prasad P, Delamora P, Larson E, Zaoutis $\mathrm{T}$ et al. Antibiotic Use in Neonatal Intensive Care Units and Adherence with Centers for Disease Control and Prevention 12 Step Campaign to Prevent Antimicrobial Resistance. Pediatr Infect Dis J. 2009 Dec; 28 (12): 1047-1051.

[16] Levy ER, Swami S, Dubois SG, Wendt R, Banerjee R. Rates and appropriateness of antimicrobial prescribing at an academic children's hospital, 2007-2010. Infect Control Hosp Epidemiol 2012; 33 (4): 346-353.

[17] Daniel M. Keller. Antibiotic Overuse Reduced Without Restricting Availability. Abstract presented at $51 \mathrm{st}$ Interscience Conference on Antimicrobial Agents and Chemotherapy (ICAAC): September 17, 2011.

[18] Akindolire AE, Tongo O, Dada-Adegbola H, Akinyinka O. Aetiology of early onset septicaemia among neonates at the University College Hospital, Ibadan. J Infect Dev Ctries 2016; 10 (12): 1338-1344. 